\title{
New design for a time-of-flight mass spectrometer with a liquid beam laser desorption ion source for the analysis of biomolecules
}

\author{
A. Charvat ${ }^{\mathrm{a})}$ and E. Lugovoj \\ MPI für Biophysikalische Chemie, Am Fassberg 11, D-37077 Göttingen and Institut für Physikalische \\ Chemie, Universität Göttingen, Tammannstrasse 6, D-37077 Göttingen, Germany \\ M. Faubel \\ MPI für Strömungsforschung, Bunsenstrasse 10, D-37073 Göttingen, Germany \\ B. Abel \\ MPI für Biophysikalische Chemie, Am Fassberg 11, D-37077 Göttingen and Institut für Physikalische \\ Chemie, Universität Göttingen, Tammannstrasse 6, D-37077 Göttingen, Germany
}

(Received 27 August 2003; accepted 4 February 2004; published 22 April 2004)

\begin{abstract}
We describe a novel liquid beam mass spectrometer, based on a recently discovered nanosecond laser desorption phenomenon, [W. Kleinekofort, J. Avdiev, and B. Brutschy, Int. J. Mass Ion. Processes 152, 135 (1996)] which allows the liquid-to-vacuum transfer, and subsequent mass analysis of pre-existing ions and ionic associates from liquid microjets of aqueous solutions. The goal of our novel technical approach is to establish a system with good mass resolution that implements improvements on critical components that make the system more reliable and easier to operate. For laser desorption pulsed dye-laser difference frequency mixing is used that provides tunable infrared light near the absorption maximum of liquid water around $3 \mu \mathrm{m}$. Different types of liquid beam glass nozzles (convergent capillary and aperture plate nozzles) are investigated and characterized. Starting from theoretical considerations of hydrodynamic drag forces on micrometer size droplets in supersonic rarefied gas flows we succeeded in capturing efficiently the liquid beam in a liquid beam recycling trap operating at the vapor pressure of liquid water. For improving the pollution resistance, the liquid jet high vacuum ion source region is spatially separated from the reflectron time-of-flight mass spectrometer (TOF-MS) working behind a gate valve in an ultrahigh vacuum environment. A simple (simulation optimized) ion optics is employed for the ion transfer from the source to the high vacuum region. This new feature is also mostly responsible for the improved mass resolution. With the present tandem-TOF-MS setup a resolution of $m / \Delta m \approx 1800$ for the low and $m / \Delta m \approx 700$ in the high mass region has been obtained for several biomolecules of different mass and complexity (amino acids, insulin, and cytochrome c). (c) 2004 American Institute of Physics. [DOI: 10.1063/1.1710704]
\end{abstract}

\section{INTRODUCTION}

The introduction of microjets of volatile liquids into high vacuum systems has recently found increasing use in pure research as well as technical applications. ${ }^{1-8}$ A new and exciting technique to study biomolecules desorbed from their natural environment in vасиo is laser induced liquid beam desorption mass spectrometry, ${ }^{8}$ recently introduced by Brutschy et al. [laser induced liquid beam ionization/desorption] (LILBID). ${ }^{1}$ Such an experimental approach enables a very soft desorption of protonated supramolecular species with an infrared laser tuned to the $\mathrm{OH}$-stretch vibration of the solvent. A significant advantage for analytic applications is that with this approach postdesorption ionization with a second laser $^{9,10}$ is not necessary which nearly completely avoids fragmentation.

In a series of publications Brutschy and co-workers have shown that this method has a great potential for the analysis of macro- and biomolecules and their complexes even in the

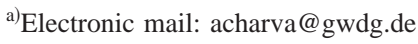

liquid phase, ${ }^{1,711-17}$ a feature that is less obvious and not straightforward for the standard and nowadays wellestablished techniques like electrospray ionization ${ }^{18}$ or matrix-assisted laser desorption/ionization (MALDI) mass spectrometry. ${ }^{19}$

In this article we describe a new LILBID- tandem mass spectrometer (MS) apparatus whose mass resolution has been increased significantly. Performance tests of different design approaches for the liquid jet nozzle source, using both, short capillary channels and convergent nozzle apertures in a diameter range between 10 and $50 \mu \mathrm{m}$ are also presented. Moreover, we have developed a liquid beam trap for the efficient removal of the sample liquid from the high vacuum ion source region by transferring and collecting the liquid water in a separate collection unit under its own vapor pressure. This simplifies the waste liquid handling or liquid recycling and by reduction of the free liquid length in the high vacuum region it reduces significantly the vapor load in the main vacuum chamber. The desorption laser system was a difference frequency mixing system which was able to generate infrared (IR) radiation between 2.6 and $2.8 \mu \mathrm{m}$ in the 


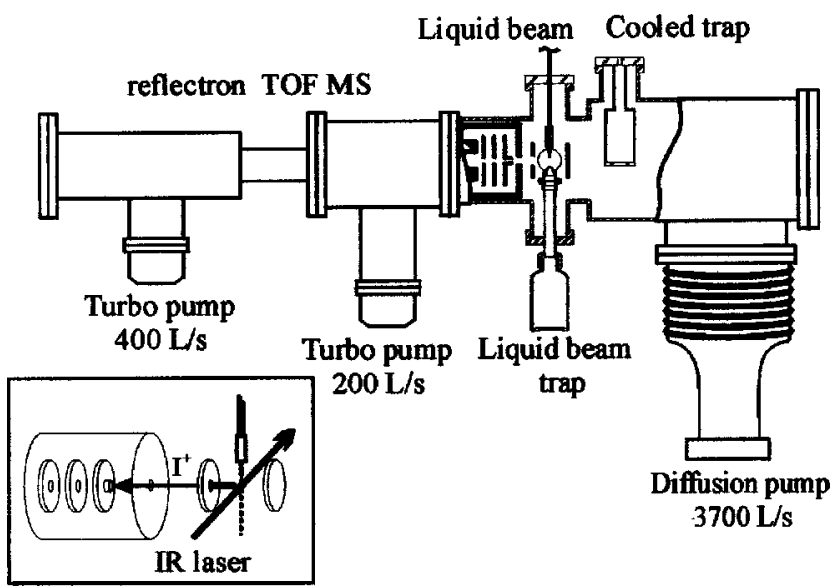

FIG. 1. Schematic picture of the experimental setup. Vacuum chamber containing the liquid beam assembly, the liquid beam trap, the reflectron timeof-flight mass spectrometer, and the vacuum pumps are shown. In the inset a more detailed view of the first extraction-acceleration ion optics is displayed.

region of the IR absorption maximum of bulk water. The laser light is tightly focused to a spot of $50 \mu \mathrm{m}$ on the liquid beam in the source vacuum region and allowed a reduction of the working pulse energy to $100-500 \mu \mathrm{J}$ by more than one order of magnitude over previous designs. Finally, an ion extraction lens system is used in the main vacuum chamber for transferring the laser desorbed ions from the liquid beam through a differential vacuum stage to a standard commercial reflectron mass spectrometer. The article is organized as follows: in Sec. II an overview of the experimental setup is given followed by the detailed description of the components of our experimental apparatus. In Sec. III we demonstrate the performance of specific parts of the setup and determine the overall performance of the tandem mass spectrometer from mass spectra of small protonated amino acids as well as proteins with masses in the range 5-12 kDa (insulin, cytochrome $c$ ). The mass resolution $(m / \Delta m)$ achievable with the present setup turned out to be much better than in previous LILBID experiments ${ }^{1}$ but still lower than in optimized MALDI-time-of-flight (TOF) experiments. ${ }^{20}$ From detailed measurements of the velocity distribution of the desorbing ions and ionic aggregates we obtain clear evidence about the origin of the moderate mass resolution in this type of experiments. The relatively broad velocity distribution and high kinetic energies of the desorbed species of several tens to hundreds of electron volts resulting from the pulsed IR laser desorption process significantly limits the mass resolution in liquid beam desorption studies. It will be shown that a tandem MS approach with an ion transfer optics and a gated ion optics can select specific portions out of the broad kinetic energy distribution. This feature turned out to be responsible for the improved mass resolution in the experiment, an approach that appears to be more efficient than the standard delayed extraction technique for this type of experiment.

\section{EXPERIMENTAL DETAILS}

Figure 1 shows a schematic diagram of the experimental apparatus used in the present study. It consists of a liquid beam, an IR laser system, and a reflectron time-of-flight mass spectrometer (reTOF-MS). A continuous liquid beam was introduced vertically into a vacuum chamber through a nozzle forming a water beam of about 10-15 $\mu \mathrm{m}$ thickness. The temperature controlled liquid water was pumped with a high pressure liquid chromatography (HPLC) pump (Gynkotek, model 300C). The flow rate was kept constant at 0.4 $\mathrm{ml} / \mathrm{min}$ throughout the experiment. The liquid beam can be trapped either in a liquid nitrogen cooled beam dump or a specially designed trap. The chamber was evacuated down to $5 \times 10^{-5}$ mbar by a $37001 \mathrm{~s}^{-1}$ diffusion pump and a cryotrap. Traveling a distance of 1-2 $\mathrm{mm}$ from the quartz nozzle exit, the liquid beam was crossed with an IR laser beam in the first ion optics. The IR wavelength is tuned into resonance with the fundamental of the $\mathrm{OH}$-stretch vibration of bulk water. In the direction perpendicular to both the laser and the water beam the produced ions are collected and accelerated towards the ion optics of the TOF spectrometer. Solutions of the amino acids with concentrations between $10^{-1}$ and $10^{-3}$ molar (M) as well as $10^{-4}$ and $10^{-5} \mathrm{M}$ solutions of insulin (from bovine pancreas, Sigma-Aldrich, 95\% purity), and cytochrome $c$ (from horse heart muscle, Acros Organics, 90\% purity) in water (LiChrosolv®, Merck) were employed in the present studies. All samples were used without further purification.

\section{A. Liquid beam production and removal in high vacuum}

The introduction and the removal of the volatile liquid jet in the mass spectrometer high vacuum region is the technically least explored and most nonstandard part of the experiment. One of the most critical components of the system is the liquid beam source. In a series of experiments we succeeded in a significant improvement of the nozzle design. A new convergent quartz nozzle is found to result in a much improved long time stability of the beam generation as opposed to the capillary channel model or the metal aperture nozzle used in previous experiments. A schematic drawing of the liquid beam nozzle-liquid beam trap assembly is shown in Fig. 2(a). The convergent quartz glass nozzle (Microliquids $\mathrm{GmbH}$ ) is fabricated from a round-capillary quartz tube $[1 \mathrm{~mm}$ inner diameter (i.d.) $\times 3 \mathrm{~mm}$ outer diameter (o.d.)]. The tube is pulled-out over an oxygen-hydrogen flame, cut at the thinnest part and polished. Apertures in the range between 10 and $15 \mu \mathrm{m}$ diameter are typical for the present type of experiments. If required, the nozzles can be coated with a hard metallic coating. The $20 \mathrm{~mm}$ long nozzle is fixed and tightened in a custom-made poly-ether-etherketone (PEEK) holder and the high pressure solvent is provided with a HPLC pump connected to the nozzle mount via a stainless steel capillary tubing $(0.75 \mathrm{~mm}$ i.d. $\times 1 / 16 \mathrm{in}$. o.d.). A photograph of the working nozzle is shown in Fig. 2(b). The transition between the stable liquid beam and the droplet region may be recognized. We want to emphasized here that the present type of nozzle produces a much more stable beam than the standard metal aperture nozzles. Also - in direct comparison with the latter-the quartz capillary nozzles have a much longer lifetime because they 


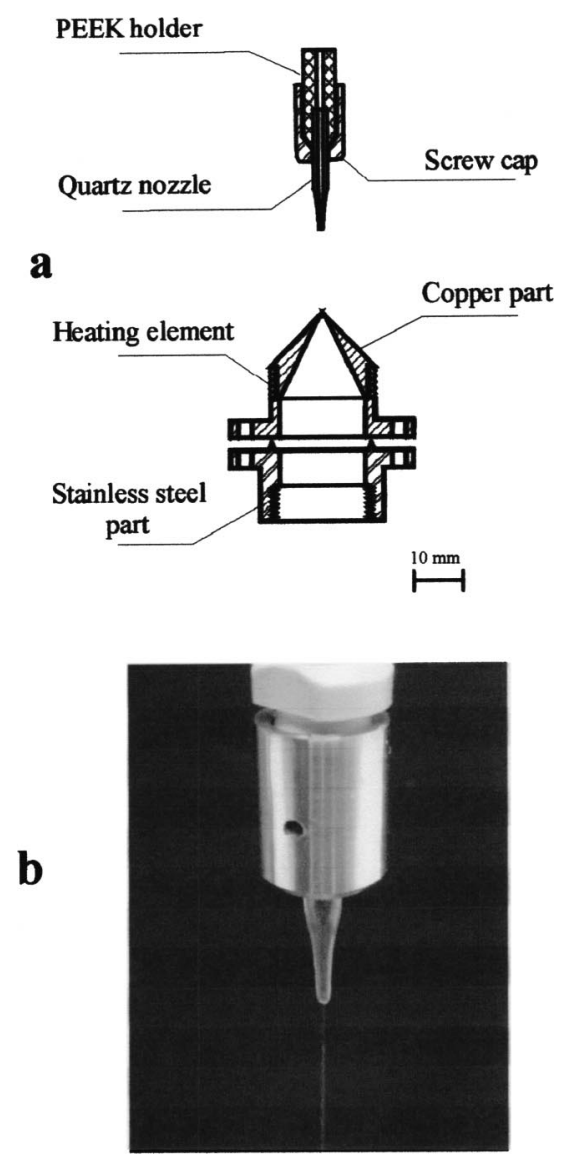

FIG. 2. Schematic view of a quartz nozzle-liquid beam trap assembly (a) and photograph of a quartz nozzle operating with a $15 \mu \mathrm{m}$ liquid beam (b). The quartz nozzle is fixed and tightened in the PEEK inlet with a stainless steel screw-cap. The stable liquid beam disintegrates after few millimeters downstream into droplets. This transition can be recognized in the change of the color of the beam in the photograph.

hardly suffer from any corrosion or damage due to high pressure deformation.

For water, with vapor pressures in the 10 mbar range, the free vacuum surface evaporation leads to large liquid surface ablation rates on the order of $1-10 \mathrm{~mm} / \mathrm{s}$ in spite of the very efficient cooling rates of $10^{5} \mathrm{~K} / \mathrm{s}$ in the initial section of the liquid jet. For a typical jet of $10 \mu \mathrm{m}$ diameter at $293 \mathrm{~K}$ the direct water vapor evaporation into the chamber high vacuum is on the order of $0.35 \mathrm{mbar}^{1} \mathrm{~s}^{-1}$ over the first $4 \mathrm{~mm}$ length of the cylindrical liquid jet. Although, a major part of the injected liquid can be removed with a cryotrap or properly chosen beam dump device, the initially evaporated gas has to be removed from the experimental chamber by rather large vacuum pumps. Typically, several $10^{4} \mathrm{l} / \mathrm{s}$ pumping speed are required for keeping a vacuum in the $10^{-5} \mathrm{mbar}$ range. If not trapped properly the supercooled liquid is forming ice deposits and rapidly growing fine needles of ice on adjacent vacuum chamber walls which can result in obstructions of the ion emission region.

We demonstrate here a new beam trap construction for removing the liquid jet from the high vacuum chamber and for collecting the liquid under its own vapor pressure. With properly chosen device dimensions the gas stream out of the beam trap can be minimized, and a stable liquid jet can be operated without applying a second vacuum system or a

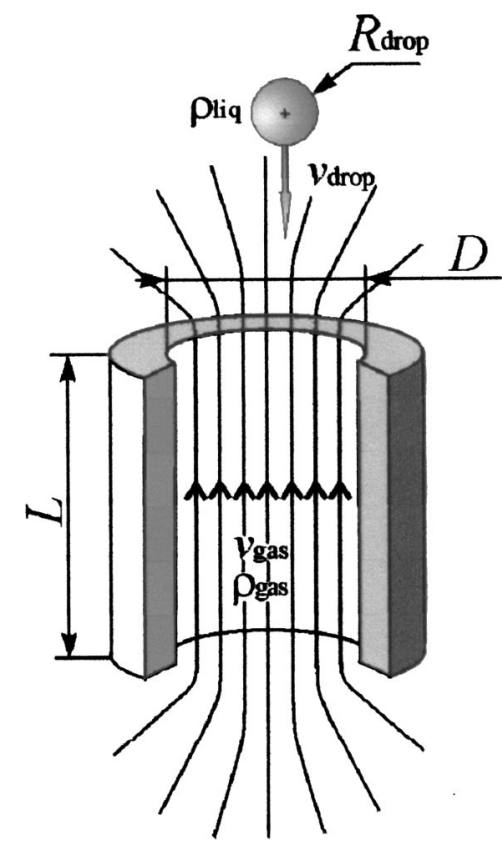

FIG. 3. Entrance channel of a skimmer of the liquid beam trap.

cooling device to the beam trap. The trap operation depends crucially on the hydrodynamic interaction between the incoming liquid jet and outward streaming vapor gas in the entrance channel of the liquid trap. After emerging from the nozzle the liquid beam first contracts somewhat ${ }^{4}$ and in turn forms a continuous cylindrical jet (of 10-30 $\mu$ m diameter) over a distance of several millimeters until it finally breaks up into droplets. In the appendix we outline a simple dynamic gas flow model and calculate the hydrodynamic friction experienced by a liquid droplet of radius $R_{\text {drop }}$ and velocity $\nu_{\text {drop }}$ with the gas backstreaming through the entrance channel of the trap (Fig. 3). According to this model, the upper limits for the channel length $L_{\max }$ and the diameter $D_{\max }$ of the entrance channel can be estimated [see Eqs. (A3) and (A7) of the Appendix].

In practice, however, the diameter $D$ is mainly chosen such that the vacuum in the main chamber can be handled. $D$ in this case is the diameter of the "artificial leak" and the leak rate strongly depends upon this parameter. Hence, $D$ as small as possible should be chosen. As the leak rate is inversely proportional to the channel length $L$ a larger channel length appears to be an advantage in terms of the achievable vacuum. However, the length $L$ must stay well below the maximum length $L_{\max }$ for the droplets to pass the entrance channel of the trap. Taking into account these criteria for a stream of $10 \mu \mathrm{m}$ diameter droplets, entrance orifices in the range of $0.1<L<0.5 \mathrm{~mm}$ and $0.05<D<0.1 \mathrm{~mm}$ were employed for a stable operation of the liquid beam source and a good vacuum (minimized backstream) in the main chamber.

The beam trap consists of two main parts [Fig. 2(a)]. The upper part is made of copper and has a conical profile with an angle of vertex of $90^{\circ}$ and a diameter of the entrance orifice of $0.1 \mathrm{~mm}$. The channel length was $0.1 \mathrm{~mm}$. Such a profile may help to prevent the formation of icicles when the supercooled water beam in vacuum touches the trap surface during the initial adjustments. For the same purposes the trap 
is equipped with a resistive heating element (Thermocoax, $25 \mathrm{~W}$ ). Thick walls of the upper part of the trap provide good heat transfer to the tip of the copper cone. The second part of the trap is made of stainless steel. An upper knife-edge surface serves two purposes: it provides a vacuum sealing between both parts of the trap when they are fastened by screws, and forms a rather broad gap between the two parts of the trap and ensures efficient thermal decoupling of both pieces. The skimmer device in our apparatus was connected to a glass reservoir via a stainless steel tube $(14 \mathrm{~mm}$ i.d.) equipped with a standard vacuum flange. The whole assembly could be translated and adjusted in the horizontal plane in two independent perpendicular directions.

\section{B. Electrostatic collection of desorbed ions from the liquid beam source}

In the direction perpendicular to both the laser and the water beam (see Fig. 1) the desorbed ions are accelerated and collimated in an ion optics with a static electric field (typically $80 \mathrm{~V} / \mathrm{cm}$ ) through two differentially pumped vacuum chambers containing the ion optics of the TOF spectrometer. The field free drift region before entering the TOF ion optics was $35 \mathrm{~mm}$.

In the inset of Fig. 1 a schematic view of the ion extraction-acceleration optics together with the acceleration region of the mass spectrometer is displayed. Such a construction enabled us to couple the liquid beam ion source to a commercially available reTOF-MS (Kaesdorf, type RTF10). The liquid jet vacuum ion source region is spatially separated from the reflectron time-of-flight mass spectrometer working behind a sliding valve (open only during experiments) in a ultrahigh vacuum environment. No extra valve for protection of the microchannel plate detector was required when the main chamber with the liquid beam assembly was at atmospheric pressure. The ion extractionacceleration optics consists of three electrodes. The repeller and extraction electrodes are polished 1-mm-thick stainlesssteel sheets with a diameter of $40 \mathrm{~mm}$. The extraction electrode had a $10 \mathrm{~mm}$ central hole. The electrically isolated plates were mounted on two rods $(\oslash 2 \mathrm{~mm})$ with variable separation. Finally, the third electrode (kept at ground potential) was fixed on the entrance side of the mass spectrometer directly above the sliding valve. The $2 \mathrm{~mm}$ aperture served simultaneously as a gas conductance barrier between the ion source and the acceleration region of the MS. The working parameters of the ion optics can be found by simulating ion trajectories with the SIMION program. ${ }^{21}$ By applying static (or pulsed) voltages to the electrodes of the optics collimation and acceleration of the ions towards the main ion acceleration region of the reTOF was achieved. In Fig. 4 a potential landscape with calculated ion trajectories is depicted for the ion optics used in the present experiments. The simulation has been done for a point ion source centered between repeller and extraction electrode, whose separation was $2.5 \mathrm{~cm}$ and respective voltages were set to $U_{1}=500 \mathrm{~V}$ and $U_{2}$ $=390 \mathrm{~V}$. The initial kinetic energy of singly charged ions with mass of $100 \mathrm{amu}$ was set to $E_{K}=0.5 \mathrm{eV}$. The distance between extraction electrode and the third electrode was 1

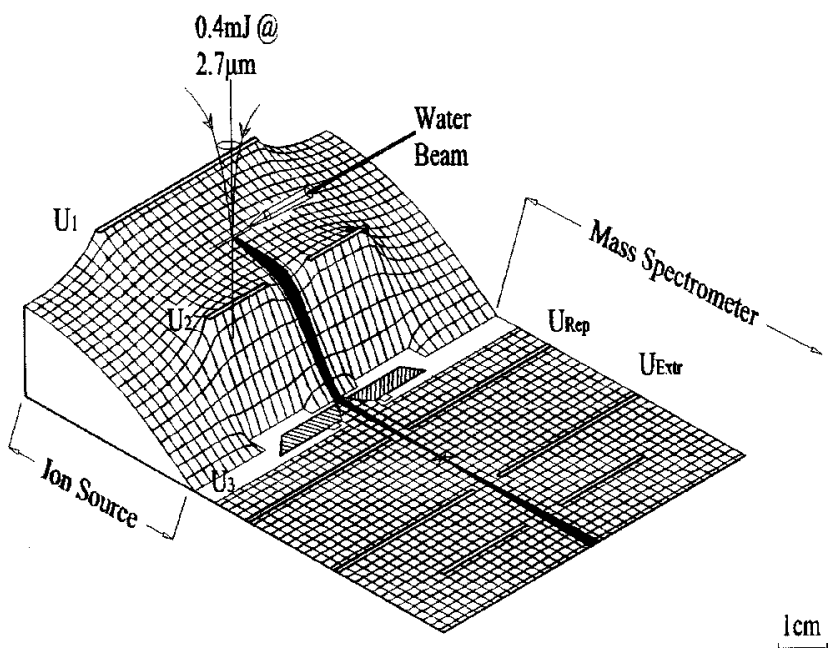

FIG. 4. Potential contours with calculated ion trajectories in the extractionacceleration ion optics region for the repeller and extraction electrode voltages set to $U_{1}=500 \mathrm{~V}$ and $U_{2}=390 \mathrm{~V}$, respectively. The other electrode and the electrode of mass spectrometer are grounded. The positively charged ions with mass $100 \mathrm{amu}$ and initial kinetic energy of $0.5 \mathrm{eV}$ are collected and focused in the ion optics of the mass spectrometer.

$\mathrm{cm}$. Since such a simple simulation does not take into account the influence of the liquid beam nozzle assembly on the electric field between electrodes, in practice, the voltages had to be adjusted somewhat in order to obtain the desired collimating effect. We even found out that in many cases only a repeller voltage between 50 and $200 \mathrm{~V}$ was sufficient to efficiently collect the ions and to record mass spectra with a good signal-to-noise ratio. The other two electrodes and the nozzle were kept at ground potential in this case.

\section{Time-of-flight mass spectrometer and data acquisition}

The three electrode optics of the reflectron TOF mass spectrometer was set to fulfill the Wiley-McLaren criterion ${ }^{22}$ and pulsed (up to $10 \mathrm{kV}$ ) with two commercial high voltage switches (Behlke, HTS121-03). The ion optics also worked as a gate for the selection of different masses (monomers, dimers) from the desorption process, because of the slightly different flight times for ions with different masses and/or genesis. In order to record the whole mass spectrum, the delay between the desorption and trigger pulse for the ion optics was varied (typically between 5 and $20 \mu$ s). The ions were then steered and focused by a set of vertical and horizontal deflectors and an einzel lens. After traveling about 600 $\mathrm{mm}$ in the field free region, the ions were reversed by the ion reflector tilted about $5^{\circ}$ off the beam axis $(1050 \mathrm{~mm}$ total field free region). During experiments the pressure in the acceleration region and in the drift tube of the mass spectrometer was $6 \times 10^{-6} \mathrm{mbar}$ and $2 \times 10^{-7}$ mbar, respectively. After passing the ion mirror and the second drift region of the spectrometer the ions were postaccelerated in front of the multichannel plate detector to $10 \mathrm{kV}$. The signals were amplified, visualized with a $200 \mathrm{MHz}$ digital oscilloscope (Tektronix, model 2440) and recorded with a $150 \mathrm{MHz}(500 \mathrm{M}$ samples's) eight-bit digitizer card (Acqiris ${ }^{\mathrm{TM}}$, model DP105) in a computer using LABVIEWTM. MS signals up to $260 \mu$ s 
with a maximum resolution of 2 ns could be recorded. Typically 100-200 individual single-shot spectra were accumulated and their summed average is presented in the figures. A digital delay generator (Stanford Research Systems, model DG535) controlled the timing of the IR laser and the ion optics.

\section{Tunable IR laser system and laser pulse intensity in the focus}

The IR laser beam was generated via difference frequency generation in a $\mathrm{LiNbO}_{3}$ crystal (INRAD ${ }^{\mathrm{TM}}$, $\mathrm{Au}-$ totracker III) from the fundamental of a pulsed Nd:yttritiumaluminum-garnet (YAG) laser (Continuum, NY81C-20, pulse length $\sim 10 \mathrm{~ns}$, repetition rate $20 \mathrm{~Hz}$ ) and the $766 \mathrm{~nm}$ output of a pulsed dye laser (Lambda Physik, Scanmate, Exciton LDS 765 dye solution in methanol). The spatial laser pulse profiles in the far-field were carefully optimized and overlapped resulting in a good beam profile of the IR radiation. The IR output energy was between 0.5 and $0.7 \mathrm{~mJ} /$ pulse. The infrared wavelength was separated from the fundamentals with a 4-mm-thick germanium plate at the Brewster angle and the IR beam was then expanded and tightly focused onto the water beam with a three lens system consisting of an expanding telescope (3:1) and a planoconvex focusing lens with a focal length of $150 \mathrm{~mm}$. For beam steering and focusing gold coated mirrors, $\mathrm{CaF}_{2}$ lenses and $\mathrm{CaF}_{2}$ entrance window were used. The transmission coefficient of the optical system was approximately 0.6. From "knife-edge" measurements ${ }^{23}$ we determined the focal spot size of the beam diameter $\left(1 / e^{2}\right)$ to be about $50-60 \mu \mathrm{m}$. Assuming $10 \mathrm{~ns}$ IR pulses, the intensity in the focal plane has been estimated to be on the order of $10^{8} \mathrm{~W} \mathrm{~cm}^{-2}$. The laser wavelength for the desorption experiments presented in this article was set to $2.735 \mu \mathrm{m}$.

\section{PERFORMANCE AND DISCUSSION}

In this section we give the specifications of the components of our setup and a thorough discussion of the performance.

\section{A. Nozzle flow performance}

In the following section we characterize the convergent channel nozzles (with orifice diameters of 15 and $24 \mu \mathrm{m}$ ) and compare them to the flat plate nozzles with a long parallel channel whose length is equal to the thickness of the plate (16 $\mu \mathrm{m}$ diameter and $1.5 \mathrm{~mm}$ long channel). For all of them we have measured the volume flow of water and ethanol as a function of pressure (both controlled via a commercial HPLC pump) at room temperature. For reliable measurements a non-negligible resistance of the steel capillary connecting the HPLC pump and the nozzle holder and of a micropore filter had to be taken into account. Therefore, the dependence of volume flow on the pressure through the flow line without the nozzle was determined first for water and ethanol and subtracted from the measurements. The experimental error of the volume flow determination did not exceed $2 \%$. The accuracy of the HPLC pressure detection was \pm 0.5 bar.
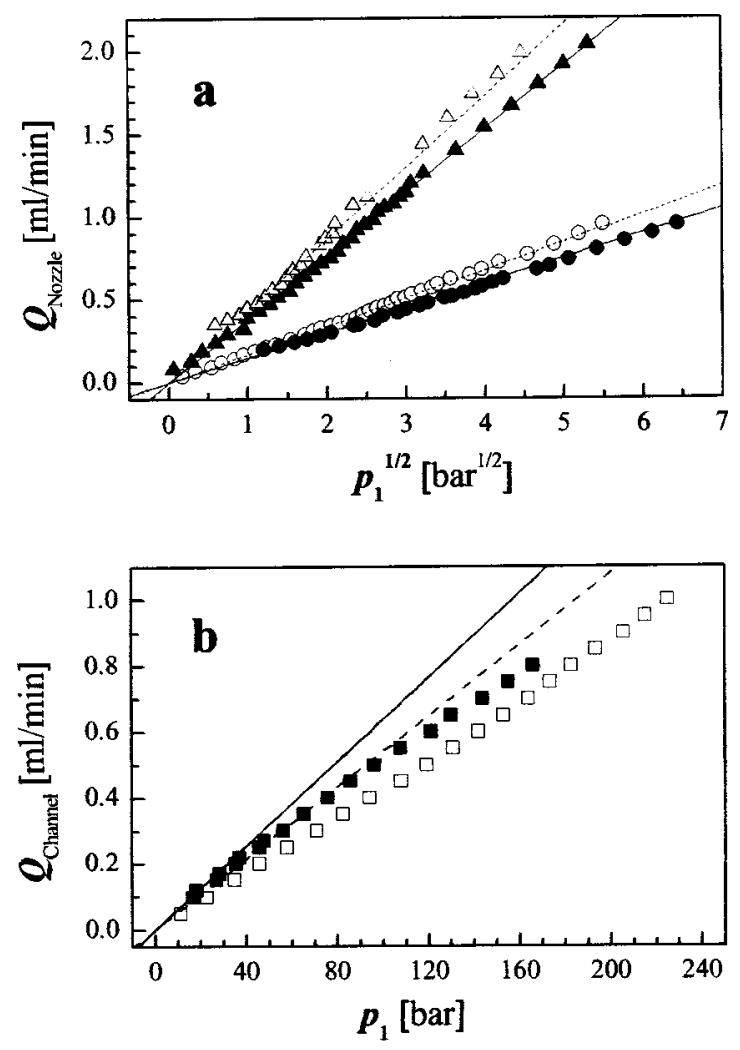

FIG. 5. Flow rates as a function of the pressure for (a) two quartz capillary nozzles: $\oslash 16 \mu \mathrm{m}-\mathrm{H}_{2} \mathrm{O}, \bigcirc \mathrm{EtOH} ; \oslash 24 \mu \mathrm{m}-\boldsymbol{\Delta} \mathrm{H}_{2} \mathrm{O}, \triangle \mathrm{EtOH}$, and (b) glass channel plates $(\oslash 16 \mu \mathrm{m}$, channel length $1.5 \mathrm{~mm})$ : $\square \mathrm{H}_{2} \mathrm{O}, \square \mathrm{EtOH}$. Solid and dashed lines represent theoretical predictions according to Eqs. (1) and (2).

Results of these measurements are presented in Fig. 5. For the convergent nozzles [Fig. 5(a)] the volume flow increases with the square root of the pressure, whereas for the plate nozzle a linear dependence on the pressure is observed [Fig. 5(b)]. It suggests that the flow may be described by the Bernoulli equation (nonviscous limit) in the former and by the Hagen-Poisseuille equation in the latter case. Namely, for the convergent nozzle with the diameter $D_{N}$ we obtain for the volume flow rate $Q$ :

$$
Q_{\text {Nozzle }}=\pi\left(\frac{D_{N}}{2}\right)^{2} \sqrt{\frac{2}{\rho}} \times \sqrt{p_{1}},
$$

where $\rho$ is the density of the liquid and $p_{1}$ is the backing pressure of the liquid.

In the case of convergent nozzle the flow rate does not depend on the viscosity of the liquid but depends on the density of the liquid. At room temperature $\rho_{\mathrm{H}_{2} \mathrm{O}}$ $=1000 \mathrm{~kg} \mathrm{~m}^{-3}$ and $\rho_{\mathrm{EtOH}}=791 \mathrm{~kg} \mathrm{~m}^{-3},{ }^{24}$ which should result in a smaller slope of the $Q_{\text {Nozzle }}$ vs $\sqrt{p_{1}}$ dependence for water as opposed to ethanol. Indeed, such a behavior is observed in the experiment and experimental values and predictions according to Eq. (1) are in good agreement.

In the case of a long parallel channel the flow rate can be described by Eq. (2):

$$
Q_{\text {Channel }}=\frac{\pi}{128} \frac{D_{C}^{4}}{\eta L_{C}} p_{1},
$$


with $p_{1}$ being the pressure at the inlet of the plate (the pressure at the outlet side being negligible small), $\eta$ is the dynamical viscosity of the liquid, $L_{C}$ is the length of the channel, and $D_{C}$ is its diameter. Since the viscosity of water at room temperature is slightly lower than that of ethanol ${ }^{24}$ the slope of the $Q_{\text {Channel }}$ vs $p_{1}$ curve is expected to be somewhat larger for water in comparison with ethanol. The experimental data shown in Fig. 5(b) are in reasonable-although not perfect-agreement with the predicted behavior. The remaining small deviations between theory and experiment may be attributed to turbulences at the entrance of the nozzle causing deviations from the ideal flow assumed in Eq. (2). As expected, we found out that the agreement between experiment and theory improves with increasing channel length.

\section{B. Performance of the liquid beam skimmer trap}

One of the important questions is how much the evaporation of the liquid in our source-trap system affects the pressure in the vacuum chamber under typical experimental conditions. The pressure in the high vacuum chamber was monitored with an ionization vacuum gauge (Leybold, Ionivac). Two sets of measurements were carried out, for water and for ethanol liquid beams, respectively. In the first set, the dependence of the background pressure on distance between nozzle and skimmer tip of the beam trap was measured. In the second, the background pressure as a function of the temperature of the liquid in the reservoir connected to the trap was detected. The results of these measurements are presented in Fig. 6(a). After correction for the relative ionization gauge sensitivities of ethanol and water vapor a similar pressure rise is observed as a function of the nozzle-totrap distance.

Evaporation rates for both ethanol and liquid water as a function of the length of the free liquid jet in the high vacuum gap between nozzle and trap entrance have also been estimated employing a simple one dimensional model. ${ }^{2}$ The results of the calculation are given in Fig. 6(b) for a liquid jet diameter of $11 \mu \mathrm{m}$ with flow rate of $0.3 \mathrm{ml} / \mathrm{min}$ (liquid beam flow speed of $23 \mathrm{~m} / \mathrm{s}$ ), and a nozzle temperature of $23^{\circ} \mathrm{C}$. Values of the enthalpies of vaporization, specific heats, and temperature dependent vapor pressures for the two liquids used in the simulation have been taken from Ref. 24. If we consider our known effective pumping speed of $10^{4} 1 \mathrm{~s}^{-1}$, the model predictions for the evaporation rates as a function of the jet length are consistent with the measurements of the pressure rise for ethanol and water at $23^{\circ} \mathrm{C}$ in Fig. 6(a).

The contribution of the back streaming gas from the room temperature liquid in the trap through the 0.1-mm-diam skimmer entrance channel was found to be approximately one order of magnitude smaller than the contribution from the direct beam evaporation. It is also interesting to compare the performances of such a liquid beam trap with a standard liquid nitrogen cooling trap $(5 \mathrm{~cm}$ diameter, $50 \mathrm{~cm}$ length) which was placed about $15 \mathrm{~cm}$ downstream from the nozzle and collinearly to the water beam. In that case the background pressure was measured to be $(6-8) \times 10^{-5} \mathrm{mbar}$ which was almost two times higher than the pressure achieved with the new skimmer trap.

As a concluding remark, we note that heating of the
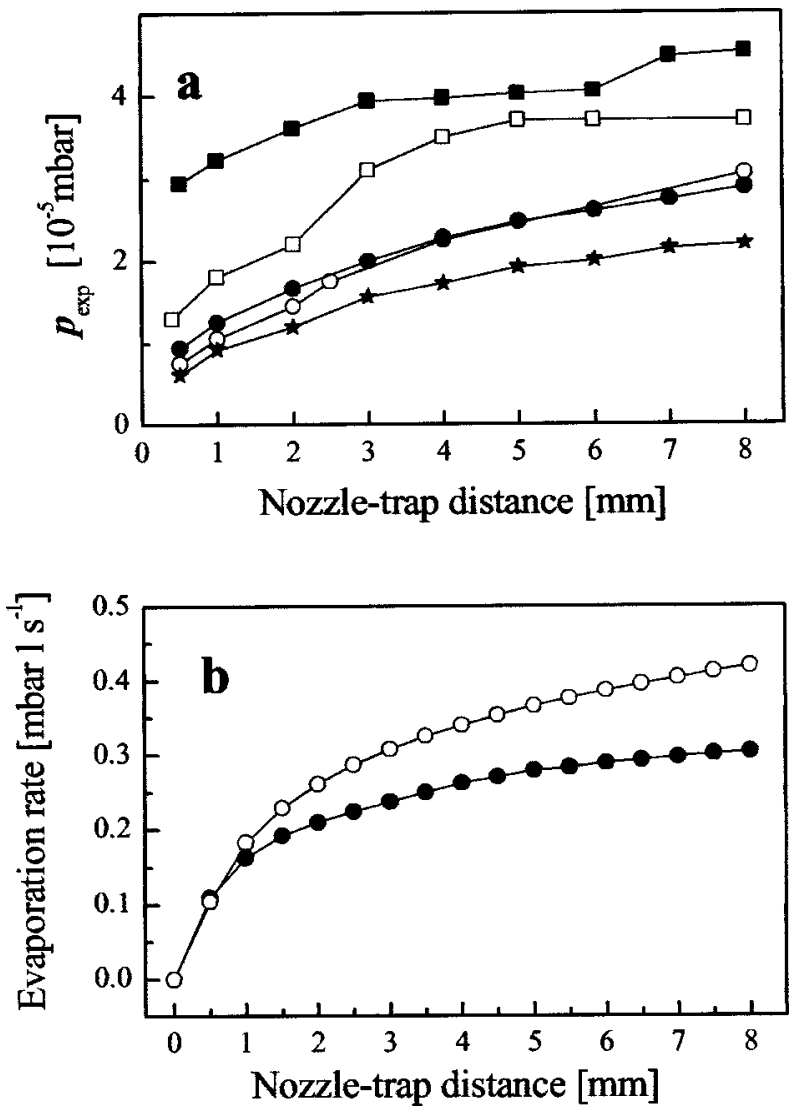

FIG. 6. (a) Background pressure dependence on the distance between nozzle and skimmer and for different temperatures of the reservoir $T_{\text {reservoir }}$ done with a quartz nozzle $\oslash 17 \mu \mathrm{m}$ (liquid beam $\oslash 11 \mu \mathrm{m}$ ) and trap entrance $\oslash 0.1 \mathrm{~mm}$. Flow rate of $0.3 \mathrm{ml} / \mathrm{min}$ and the temperature of the liquid in the nozzle $T_{\text {nozzle }}=23^{\circ} \mathrm{C}$. $\square \mathrm{EtOH}, T_{\text {reservoir }}=23^{\circ} \mathrm{C}, \square \mathrm{H}_{2} \mathrm{O}$, $T_{\text {reservoir }}=23^{\circ} \mathrm{C} ; \star \mathrm{EtOH}, T_{\text {reservoir }}=-196{ }^{\circ} \mathrm{C} ; \bullet \mathrm{EtOH}, T_{\text {reservoir }}=0{ }^{\circ} \mathrm{C}$, and $\bigcirc \mathrm{H}_{2} \mathrm{O}, T_{\text {reservoir }}=0{ }^{\circ} \mathrm{C}$. (b) Theoretical calculation of the evaporation from the liquid beam $(\oslash 11 \mu \mathrm{m})$ with $T_{\text {nozzle }}=23{ }^{\circ} \mathrm{C} ; \bigcirc \mathrm{H}_{2} \mathrm{O}, \bullet \mathrm{EtOH}$.

skimmer was necessary only during the initial adjustment of the liquid beam position and only when water was used as the liquid. After the system has been aligned and the water beam passed through the entrance orifice stable operation over several hours could be achieved without heating.

\section{Mass resolution of the tandem mass spectrometer}

The mass resolution that can be achieved in our experiment in the low mass region is illustrated in Fig. 7. Time-offlight mass spectra of the protonated lysine dimer [molecular weight $(\mathrm{MW})=293.4]$ is depicted there. The $0.1 \mathrm{M}$ solution of lysine hydrochloride in water at $p \mathrm{H} \simeq 6-7$ was kept at room temperature. The full width at half maximum (FWHM) mass resolution in this case is $1.9 \times 10^{3}$. In order to achieve this resolution a careful control of the voltages (ion reflector, ion focusing/steering elements) in the mass spectrometer, as well as the time delay, $T_{D}$, which is the time between desorption event and application of the high voltage to the ion extraction optics of the mass spectrometer, was necessary. Bovine insulin ( $\mathrm{MW}=5733.6)$ and cytochrome $c$ (MW $=12228.4$ ) are widely used benchmark substances for testing performances of mass spectrometers (resolving power, 


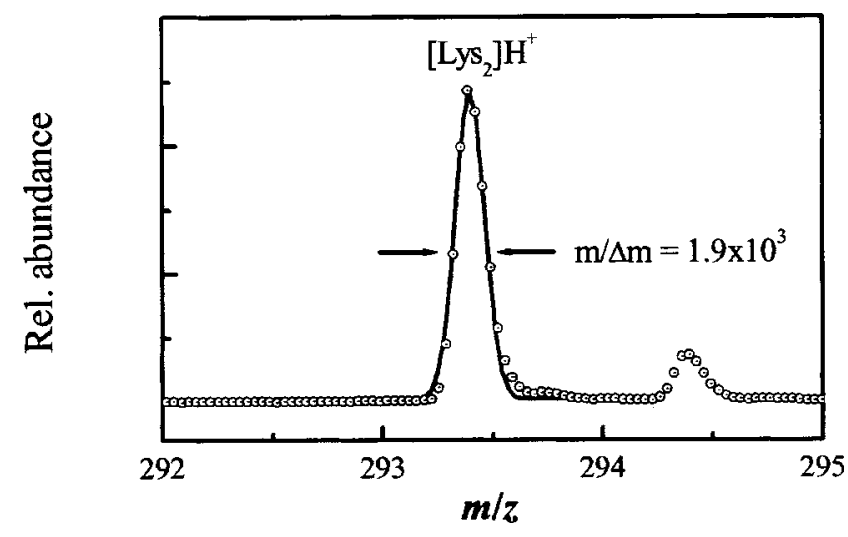

FIG. 7. Time-of-flight mass spectra of the protonated lysine dimer $(0.1 \mathrm{M}$, $p \mathrm{H}=6-7)$. The FWHM mass resolution achieved in this experiment was $1.9 \times 10^{3}$. The time delay $T_{D}=6.33 \mu \mathrm{s}$. The solid curve provides a Gauss fit to the experimental data. The small secondary peak in the spectrum is due to the ${ }^{13} \mathrm{C}$ isotope.

mass accuracy determination) in the mass region 5000$15000 \mathrm{Da}$. In the present case we are particularly interested in the achievable mass resolution. The mass spectra of both molecules obtained by laser induced liquid beam desorption are shown in Fig. 8. The mass resolution in the high mass region was determined to be 460 in the case of insulin [Fig. 8(a)] and about 720 for cytochrome $c$ [Fig. 8(b)]. The resolution is found to be a factor of 3-4 lower than in the case of the lysine dimer (Fig. 7). Nevertheless the obtained mass resolution is significantly higher $(5-10 \times)$ than published in liquid beam desorption experiments so far. It is difficult to
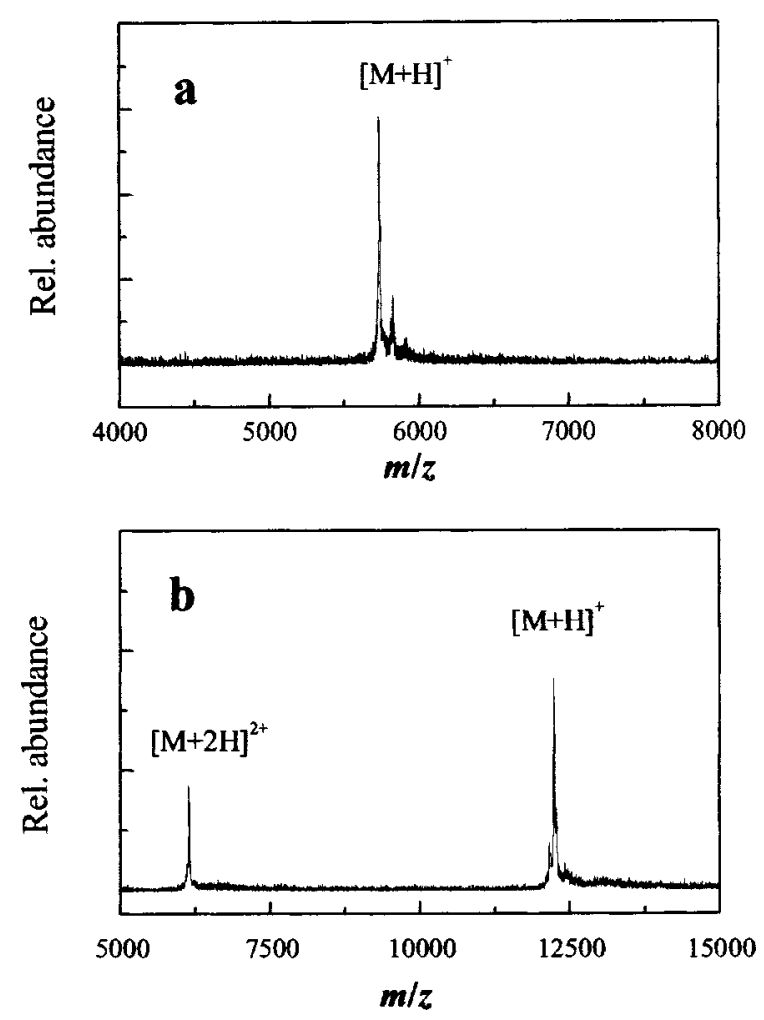

FIG. 8. Time-of-flight mass spectra of bovine insulin $\left(3 \times 10^{-5} \mathrm{M}, p \mathrm{H}\right.$ $=2-3)$ (a) and cytochrome $c\left(10^{-4} \mathrm{M}, p \mathrm{H}=6-7\right)$ (b) with FWHM mass resolution of 460 and 720 , respectively. The time delay $T_{D}$ in these cases was set to $11.4 \mu$ s (insulin) and $6.5 \mu \mathrm{s}$ (cytochrome $c$ ). directly compare with results from MALDI TOF-MS. Because of different instrumental parameters a large variation of results can be found in the literature for molecules of similar complexity. A resolution of 12500 for bovine insulin has been reported with a $6.4 \mathrm{~m}$ MALDI reflectron TOF-MS. ${ }^{20}$ In other MALDI experiments with a linear TOF-MS, the resolution achieved was roughly 80 (1 m TOF instrument), ${ }^{25} 1000$ in the first experiments employing delayed extraction, ${ }^{26}$ and about 7000 with a postsource pulsefocusing technique. ${ }^{27}$ Mass spectra of cytochrome $c$ have also been recorded recently by Hillenkamp and co-workers. ${ }^{28,29}$ Their resolution in delayed extraction mode with a reflectron TOF instrument (3.5 m drift length) was reported to be 1500 and 620 with Er:YAG $(\lambda=2.94 \mu \mathrm{m})$ and TEA- $\mathrm{CO}_{2}$ laser desorption, respectively. The same resolution has been achieved with a linear TOF-MS in recent ultraviolet-MALDI experiments with time-lag focusing using a nitrogen laser. In particular, Brown and Lennon published a value of 1025 obtained with a $2.3 \mathrm{~m}$ spectrometer, ${ }^{30}$ whereas Whittal and Li obtained a somewhat smaller resolution of 800 with a $1 \mathrm{~m}$ instrument. ${ }^{26}$

\section{Ion gate applications and measurements of ion drift times}

As the final velocity of the ions in the first extractionacceleration ion optics depends naturally on their mass-tocharge ratio, the time needed for ions to reach the extraction region of the mass spectrometer will be different for different ion masses provided the initial velocity and the genesis of the ions are similar. The extraction ion optics of the mass spectrometer can therefore act as an ion gate, that transmits only ions of specific masses. An illustration of this feature of the setup is shown in Fig. 9. At a time delay of $T_{D}$ $=5.03 \mu \mathrm{s}$ only the protonated lysine monomer appears in the mass spectrum [Fig. 9(a)], whereas at $T_{D}=6.33 \mu$ s the protonated lysine dimer can be detected [Fig. 9(b)]. The small lines around $200 \mathrm{amu}$ correspond to protonated water clusters $\left[\mathrm{H}_{2} \mathrm{O}\right]_{n} \mathrm{H}^{+}$. The monomer signal appears to be almost negligible at that time delay. This setup is therefore well suited for background free photofragment spectroscopy on well defined and selected molecular complexes (dimer versus monomer, for instance), experiments which are planned in our laboratory.

The measured delay times $T_{D}$ are a measure of the flight time in the drift region after desorption and acceleration in a static field. As shown in Ref. 8 they also allow for a determination of the average initial velocity $\nu_{0}$ of the desorbing species. A calculation of the flight time in the ion optics and the drift region depends upon the initial velocity $\nu_{0}$ of the cationic species in the initial phase of the explosion. In Fig. 10 experimental points and calculated delay times $T_{D}$ for arginine monomers and multimers are compared for a repeller voltage of $50 \mathrm{~V}$. A fit of $\nu_{0}$ to the experimental data enabled a rough estimate of $\nu_{0}=2000-3000 \mathrm{~ms}^{-1}$, a value that is significantly above that determined recently for lysine for other desorption parameters. ${ }^{8}$ This reflects our experience in this type of experiment, namely, that the kinetic energy distribution of the desorbed species strongly depends upon 

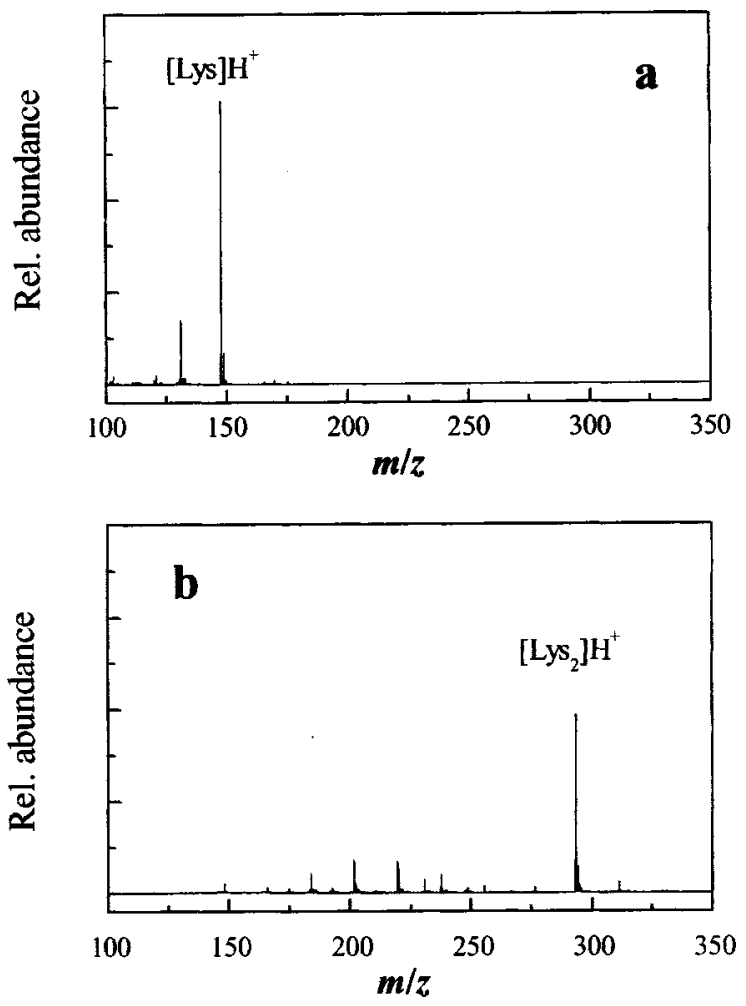

FIG. 9. Mass spectra of protonated lysine monomer (a) and protonated lysine dimer (b) from a $0.1 \mathrm{M}$ concentration of aqueous solution $(p \mathrm{H}$ $=6-7)$. The time delays $T_{D}$ were 5 and $6.3 \mu$ s for the monomer and dimer, respectively.

the desorption parameters (laser power, wavelength, beam profile, etc.). Nevertheless, the gating technique described here is a powerful approach to shed more light on the initial kinetic energy distribution and the mechanism(s) of the not so well understood laser induced liquid beam desorption process.

\section{DISCUSSION}

In the present article we describe a new design for a time-of-flight mass spectrometer with a liquid beam laser desorption ion source for the analysis of biomolecules. The progress and changes in comparison to previous designs are a modification of the liquid beam nozzles and the introduc-

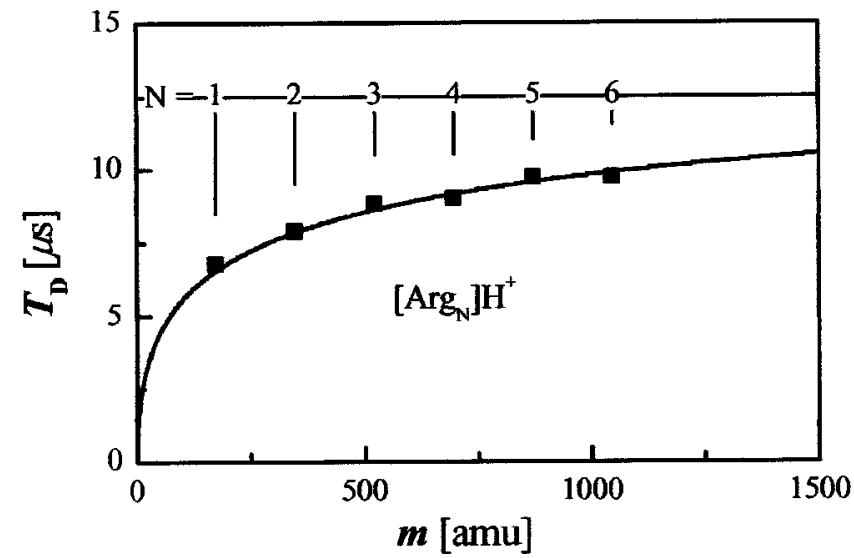

FIG. 10. Time delays $T_{D}$ adjusted for protonated arginine multimers $(N$ $=1-6)$ measured with a $0.1 \mathrm{M}$ aqueous solution $(p \mathrm{H}=6-7)$. Experimental data $(\bullet)$ are fitted with a simple model (solid line). tion of a novel beam dump which allows to collect the liquid under its own vapor pressure. The latter significantly simplifies the vacuum system. By separation of the ion injection zone from the two field acceleration region of the TOF mass spectrometer both an enhanced mass resolution of an order of magnitude as compared to earlier designs and a reduction of pressure in the TOF spectrometer is achieved. Although the advantage of the new ion transfer in terms of the increase of the mass resolution is obvious it also decreases the ion collection efficiency to some extent. The advantage of the new feature is clearly the possibility to isolate fractions of desorbed molecules for special spectroscopic applications but in order to obtain a complete mass spectrum of all desorbed molecules it will be necessary to scan the waiting time $T_{D}$ and sum up the different mass spectra at different delay times.

Although, the novel design has several significant advantages over previous designs it also has a major limitation which is not severe for a prototypical research system but which is important for an analytical system for the investigation of expensive biomolecules in very small quantities. Due to the continuous water beam and the low repetition desorption laser system $(20 \mathrm{~Hz})$ the repetition rate duty cycle is far from being optimal for the experiment. For an analytical instrument this may be an important figure of merit. This limitation of the present configuration can be overcome with high repetition laser systems which are available up to 10 $\mathrm{kHz}$ nowadays. The ultimate improvement in terms of the duty cycle of the device will be the use of a droplet source (instead of a continuous beam) that provides fast droplets on demand which are synchronized to the desorption laser. Such an extension of the present setup is under development in our laboratory at present and preliminary results will be published elsewhere. In this case the material consumption, the detection limits, as well as the duty cycle will easily meet the high standards for analytical mass spectrometers with other sources.

\section{ACKNOWLEDGMENTS}

The authors thank the Deutsche Forschungsgemeinschaft (SFB 357 "Mechanismen unimolekularer Reaktionen" and GK 782 "Spectroscopy and dynamics of molecular aggregates, coils, chains and networks"), and the Fonds der Chemischen Industrie for financial support.

\section{APPENDIX}

In the following we will briefly consider the hydrodynamic friction experienced by a liquid droplet of radius $R_{\text {drop }}$ and velocity $\nu_{\text {drop }}$ with the gas backstreaming through the entrance channel of the trap (Fig. 3). After emerging from the nozzle the liquid beam first contracts ${ }^{4}$ due to flow relaxation forces and to surface tension. A $10-30 \mu \mathrm{m}$ size jet is formed with a smooth and continuous part with a length of several hundred jet diameters, or, typically several millimeters. Due to the well known Rayleigh instability it unavoidably breaks up into droplets after several millimeters. In order to be able to calculate the proper device dimensions, i.e., the length $L$ and the diameter $D$ of the beam trap entrance channel, we 
applied a dynamic gas flow model by taking into account the perturbation of a liquid droplet with the density $\rho_{\text {liq }}$ caused by a gas stream leaving the beam trap. The deceleration of the droplet is discussed for two different regions. In region I located inside of the entrance channel of length $L$ the gas flow out of the trap resembles a piston flow and the deceleration of the incoming liquid spheres can be described with Newton's formula for laminar flow yielding an expression that limits the channel length $L$ (length of strong interaction). In region II the gas that is leaving the beam dump skimmer into the high vacuum chamber undergoes a supersonic expansion. The friction on the droplets due to this gas flow is related to the diameter D of the entrance channel (see Fig. 3).

\section{Region I: Entrance channel}

The drag force $F_{\text {drag }}$ on a liquid sphere with radius $R_{\text {drop }}$ that passes the entrance channel of the beam dump is calculated from a Newton type formula for the flow resistance

$$
F_{\text {drag }}=\frac{1}{2} c_{d} \cdot \rho_{\text {gas }} \pi R_{\text {drop }}^{2} \nu_{\text {gas }}^{2},
$$

where $c_{d}$ is the drag coefficient (e.g., for rarefied supersonic gas flows the drag coefficient $c_{d}$ of a single, rigid sphere is close to $1-2) .{ }^{31}$ The gas density $\rho_{\text {gas }}$ can be calculated from the gas vapor pressure inside the beam trap. The gas velocity is assumed to be the enthalpy limited (supersonic) flow velocity for the stagnant vapor, $\nu_{\text {gas }}=\sqrt{H_{0} / 2 m}$, with $H_{0}$ being the enthalpy of the vapor and $m$ its molecular mass.

The drag force decelerates the liquid droplet according to

$$
b_{\mathrm{drag}}=\frac{F_{\mathrm{drag}}}{\frac{4}{3} \pi R_{\mathrm{drop}}^{3} \rho_{\mathrm{liq}}},
$$

limiting the depth of penetration into the entrance channel of the beam trap to

$$
L_{\max }=\frac{\nu_{\text {drop }}^{2}}{2 b_{\text {drag }}}=\frac{4}{3} c_{d}^{-1}\left(\frac{\nu_{\text {drop }}}{\nu_{\text {gas }}}\right)^{2} \frac{\rho_{\text {liq }}}{\rho_{\text {gas }}} R_{\text {drop }} .
$$

$L_{\max }$ is the maximum length of the entrance channel that supports penetration against the Newton friction of the outflowing vapor gas with constant velocity.

\section{Region II: Supersonic radial expansion flow into the high vacuum chamber}

The supersonic gas flow out of the entrance channel into the high vacuum chamber resembles a radial isotropic expansion along the symmetry axis of the device. For a short channel length $L$ the density of the expanded gas $\rho_{\text {gas }}(z)$ decreases with the distance $z$ from the aperture of the channel according to ${ }^{32}$

$$
\rho_{\text {gas }}(z)=0.1 \rho_{\text {gas }}\left(\frac{D}{z}\right)^{2} .
$$

Here, the distance $z$ is reported with respect to $z_{0}$, where $z_{0}$ is the location of the virtual center of expansion with the gas density $\rho_{\text {gas }}\left(z_{0}\right)=\rho_{\text {gas }}$. From Eq. (A4) follows $z_{0}=D / \sqrt{10}$. To evaluate the work of the drag force $F_{\text {drag }}$ acting on the liquid droplet, Eq. (A1) has to be integrated along $z$ according to Eq. (A5):

$$
W_{\mathrm{drag}}=\int_{z_{0}}^{\infty} F_{\mathrm{drag}} d z=\frac{\pi}{2 \cdot \sqrt{10}} c_{d} D \rho_{\mathrm{gas}} R_{\mathrm{drop}}^{2} \nu_{\mathrm{gas}}^{2}
$$

This energy has to be compared with the available kinetic energy of the droplet given in Eq. (A6):

$$
E_{\text {drop }}=\frac{1}{2}\left(\frac{4}{3} \pi R_{\text {drop }}^{3} \rho_{\text {liq }}\right) \nu_{\text {drop }}^{2}
$$

The condition $E_{\text {drop }} \geqslant W_{\text {drag }}$ yields an estimate for the maximum channel diameter

$$
D_{\max }=\sqrt{10} \frac{4}{3} c_{d}^{-1}\left(\frac{\nu_{\text {drop }}}{\nu_{\text {gas }}}\right)^{2} \frac{\rho_{\text {liq }}}{\rho_{\text {gas }}} R_{\text {drop }}
$$

A comparison of Eqs. (A3) and (A7) provides a relation between $D_{\max }$ and $L_{\max }$ :

$$
D_{\max }=\sqrt{10} L_{\max } \cong 3 L_{\max } .
$$

For quantitative estimations we want to emphasize that the value of the effective drag coefficient $c_{d}$ in Eqs. (A3)(A7) for our particular situation (sequence of droplets moving in vapor stream) should be determined experimentally. For instance, we have observed that with a channel length $L=5 \mathrm{~mm}$ and $D=0.2 \mathrm{~mm}$ the incoming water droplet beam $\left(\nu_{\text {drop }}=30 \mathrm{~ms}^{-1}, R_{\text {drop }}=10 \mu \mathrm{m}, \rho_{\text {drop }}=1 \mathrm{~g} \mathrm{~cm}^{-3}\right.$ ) was deflected at pressures in the trap of approximately 100 mbar $\left(\rho_{\text {gas }} \sim 7 \times 10^{-5} \mathrm{~g} \mathrm{~cm}^{-3}, \quad \nu_{\text {gas }} \approx 300-400 \mathrm{~ms}^{-1}\right)$. According to Eq. (A3) the value of $c_{d}$ is of about 0.4 in this case.

${ }^{1}$ W. Kleinekofort, J. Avdiev, and B. Brutschy, Int. J. Mass Spectrom. Ion Processes 152, 135 (1996).

${ }^{2}$ M. Faubel, S. Schlemmer, and J. P. Toennies, Z. Phys. D: At., Mol. Clusters 10, 269 (1988).

${ }^{3}$ M. Faubel and T. Kisters, Nature (London) 339, 527 (1989).

${ }^{4}$ M. Faubel and B. Steiner, Ber. Bunsenges. Phys. Chem. 96, 1167 (1992).

${ }^{5}$ M. Faubel, Photoionization and Photodetachment (World Scientific, Singapore, 2000).

${ }^{6}$ G. Korn, A. Thoss, H. Stiel, U. Vogt, M. Richardson, M. Faubel, and T. Elsässer, Opt. Lett. 27, 866 (2002).

${ }^{7}$ B. A. M. Hansson, M. Berglund, O. Hemberg, and H. M. Hertz, Proc. SPIE 3997, 729 (2000).

${ }^{8}$ A. Charvat, E. Lougovoj, M. Faubel, and B. Abel, Eur. Phys. J. D 20, 573 (2002).

${ }^{9}$ J. Kohno, F. Mafune, and T. Kondow, J. Phys. Chem. A 105, 8939 (2001).

${ }^{10}$ T. Kondow and F. Mafune, Annu. Rev. Phys. Chem. 51, 731 (2000).

${ }^{11}$ W. Kleinekofort, A. Pfenninger, T. Plomer, C. Griesinger, and B. Brutschy, Int. J. Mass Spectrom. Ion Processes 156, 195 (1996).

${ }^{12}$ W. Kleinekofort, M. Schweizer, J. W. Engels, and B. Brutschy, Int. J. Mass Spectrom. Ion Processes 163, 1L (1997).

${ }^{13}$ A. Wattenberg, H.-D. Barth, and B. Brutschy, J. Mass Spectrom. 32, 1350 (1997).

${ }^{14}$ A. Wattenberg, H.-D. Barth, and B. Brutschy, J. Mass Spectrom. 32, 1350 (1997).

${ }^{15}$ A. Wattenberg, F. Sobott, H.-D. Barth, and B. Brutschy, Int. J. Mass. Spectrom. 203, 49 (2000)

${ }^{16}$ A. Wattenberg, F. Sobott, and B. Brutschy, Rapid Commun. Mass Spectrom. 203, 49 (2000).

${ }^{17}$ A. Wattenberg, F. Sobott, H.-D. Barth, and B. Brutschy, Int. J. Mass. Spectrom. 203, 49 (2000).

${ }^{18}$ M. Yamashita and J. B. Fenn, J. Phys. Chem. 88, 4451 (1984). 
${ }^{19}$ M. Karas and F. Hillenkamp, Anal. Chem. 60, 2299 (1988).

${ }^{20}$ R. D. Edmonson and D. H. Russel, J. Am. Soc. Mass Spectrom. 7, 995 (1996).

${ }^{21}$ D. A. Dahl, SIMION program Ver. 7.0 (Idaho National Engineering and Environmental Laboratory, 2000).

${ }^{22}$ W. C. Wiley and I. H. McLaren, Rev. Sci. Instrum. 26, 1150 (1955).

${ }^{23}$ A. E. Siegman, M. W. Sasnett, and T. F. Johnston, Jr., IEEE J. Quantum Electron. 27, 1098 (1991).

${ }^{24}$ CRC Handbook (CRC Press, Cleveland, 2000).

${ }^{25}$ G. R. Kinsel, R. D. Edmonson, and D. Russel, J. Mass Spectrom. 32, 714 (1997).
${ }^{26}$ R. M. Whittal and L. Li, Anal. Chem. 67, 1950 (1995).

${ }^{27}$ M. Amft, F. Moritz, C. Weickhardt, and J. Grotemeyer, Rapid Commun. Mass Spectrom. 12, 1897 (1998).

${ }^{28}$ S. Berkenkamp, C. Menzel, M. Karas, and F. Hillenkamp, Rapid Commun. Mass Spectrom. 11, 1399 (1997).

${ }^{29}$ C. Menzel, S. Berkenkamp, and F. Hillenkamp, Rapid Commun. Mass Spectrom. 13, 26 (1999).

${ }^{30}$ R. S. Brown and J. L. Lennon, Anal. Chem. 67, 1998 (1995).

${ }^{31}$ G. A. Bird, Molecular Gas Dynamics and the Direct Simulation of Gas Flows (Clarendon, Oxford, 1994).

${ }^{32}$ H. Pauly, Atom, Molecule and Cluster Beams I (Springer, Berlin, 2000). 